\title{
喷雾干燥可控制备生物玻璃微球及其体外生物活性研究
}

\author{
胡亚萍 ${ }^{1,2}$, 田正芳 ${ }^{2}$, 朱 敏 ${ }^{1}$, 朱铨方 ${ }^{1,2}$
}

(1. 上海理工大学 材料科学与工程学院, 上海 200093; 2. 黄冈师范学院 化学化工学院, 催化材料制备及应用湖 北省重点实验室, 湖北 438000)

摘 要: $\mathrm{CaO}-\mathrm{SiO}_{2}-\mathrm{P}_{2} \mathrm{O}_{5}$ 体系生物玻璃(Bioglass, $\mathrm{BG}$ )微球具有良好的生物活性和骨传导性, 在骨组织修复领域得到 广泛研究与应用。传统熔融法制备 BG 粉体的能耗大、粉体形貌不可控、生物活性相对较低; 溶胶-凝胶法制备 BG 粉体则需大量溶剂、制备周期长、不易量产。为快速、规模化制备形貌、粒径、化学组成可控的 BG 微球, 本研究 以水溶液为溶剂, 以正硅酸四乙酯、磷酸三乙酯、四水硝酸钻为原料, 采用喷雾干燥前驱体溶液方法制备 BG 微球, 探讨喷雾干燥过程中进气风量、前驱体溶液浓度和进料速率等工艺参数对 BG 微球粒径的影响; 前驱体溶液化学组 成对 $\mathrm{BG}$ 微球的体外诱导磷灰石沉积能力的影响。结果表明, BG 微球的粒径范围在 $40 \mu \mathrm{m}$ 以下可控, 且粒径随前 驱体溶液浓度增大而增大, 随进气风量增大而减小, 进料速率则对微球粒径影响较小。不同化学组成的 BG 微球都 具有良好的体外诱导磷灰石沉积能力, 而且随 $\mathrm{CaO}$ 含量的增加而提高。

关 键 词: 喷雾干燥; 生物玻璃; 微球; 磷灰石

中图分类号: TQ174 文献标识码: A

\section{Controllable Preparation and in Vitro Bioactivity of Bioglass Microspheres via Spray Drying Method}

\author{
HU Yaping ${ }^{1,2}$, TIAN Zhengfang ${ }^{2}$, ZHU Min $^{1}$, ZHU Yufang $^{1,2}$
}

(1. School of Materials Science and Engineering, University of Shanghai for Science and Technology, Shanghai 200093, China; 2. Hubei Key Laboratory of Processing and Application of Catalytic Materials, College of Chemical Engineering, Huanggang Normal University, Huanggang 438000, China)

\begin{abstract}
The $\mathrm{CaO}-\mathrm{SiO}_{2}-\mathrm{P}_{2} \mathrm{O}_{5}$ bioglass (BG) microspheres with good bioactivity and osteoconductivity have been extensively studied for bone tissue engineering. Traditional melting method for preparing BG powders is high energy consumption, and difficult to control the morphology of BG. While Sol-Gel technique for BG preparation requires a large amount of organic solvent, long preparation period and low mass production. For the rapid and scalable preparation of BG microspheres with controllable morphology, particle size and chemical composition, an efficient strategy of spray drying of precursor solution was proposed in this study, and the effects of process parameters including inlet air volume, precursor solution concentration and feed rate on the particle size of BG microspheres, and the effect of chemical composition on the apatite formation ability of BG microspheres in vitro were investigated. The results showed that the particle sizes of BG microspheres via spray drying could be controlled below $40 \mu \mathrm{m}$, and the particle sizes of BG microspheres increased with the concentration of precursor solution, and decreased
\end{abstract}

收稿日期：2019-12-05; 收到修改稿日期：2020-01-28

基金项目: 上海市科委地方院校能力建设专项计划(17060502400) Science and Technology Commission of Shanghai Municipality (17060502400)

作者简介: 胡亚萍(1995-), 女, 硕士研究生. E-mail: hp221727470@163.com HU Yaping (1995-), female, Master candidate. E-mail: hp221727470@163.com

通讯作者: 朱敏, 副教授. E-mail: mzhu@usst.edu.cn; 朱钰方, 教授. E-mail: zjf2412@163.com ZHU Min, associate professor. E-mail: mzhu@usst.edu.cn; ZHU Yufang, professor. E-mail: zjf2412@163.com 
with the increase of inlet air volume, but the feed rate has a slight effect on the particle size of the microspheres. On the other hand, BG microspheres with different chemical compositions had good ability to induce apatite formation in vitro, and it increased with the increase of $\mathrm{CaO}$ content. Therefore, spray drying method would be promising for rapid and scalable preparation of BG microspheres.

Key words: spray drying; bioglass; microsphere; apatite

$\mathrm{SiO}_{2}-\mathrm{CaO}-\mathrm{P}_{2} \mathrm{O}_{5}$ 体系的硅酸盐基生物玻璃 (Bioglass, BG)能与人体体液发生化学反应而与骨组 织形成界面结合 ${ }^{[1]}$ 。BG 具有优异的生物相容性、成 骨能力和降解性能，因此自 1969 年被发明以来，已 得到广泛应用和深入研究 ${ }^{[1-4]}$ 。

传统制备 BG 粉体的方法有高温熔融法、溶胶凝胶法等。高温熔融法制备 BG 粉体具有高温耗能、 混料不匀和生物活性较低等特点 ${ }^{[5-6]}$ 。因此, 为了克 服这些缺点, 研究人员提出溶胶-凝胶法, 可在室温 下成功制备出 $\mathrm{BG}$ 粉体 ${ }^{[6-9]}$ 。与高温熔融法相比, 溶 胶一凝胶法制备的 BG 粉体生物活性更好，但其存在 制备周期长、需用有机溶剂 ${ }^{[10]}$, 且块状 $B G$ 还需要 进一步研磨等缺点。

喷雾干燥是一种快速加热和冷却的连续过程。 在制备微球方面, 喷雾干燥法具有耗时短、可批量 生产、干燥过程还能方便调节微球大小和形状等优 点 ${ }^{[11-17]}$ 。例如, Yamauchi 等 ${ }^{[11]}$ 采用结构导向剂 F127、 1,2-双 (三乙氧基甲硅烷基)乙烷和正硅酸四乙酯混 合后喷雾干燥制备了具有良好球形形貌和粒径在 $0 \sim 2.5 \mu \mathrm{m}$ 的有机硅微球。Fiorilli 等 ${ }^{[12]}$ 采用正硅酸四 乙酯为原料预水解后结合三嵌段表面活性剂 P123 进行喷雾干燥制备出粒径为 $2 \sim 5 \mu \mathrm{m}$, 孔径约 $8 \mathrm{~nm}$ 的二氧化硅微球。近年来, 研究人员采用喷雾干燥 法替代传统工艺制备 BG 粉体。例如, Shih 等 ${ }^{[18]}$ 采 用溶胶一凝胶法结合喷雾干燥可快速制备平均孔径 为 $6 \mathrm{~nm}$, 比表面积为 $260 \mathrm{~cm}^{2} / \mathrm{g}$ 的 BG 微球。Ostomel 等 ${ }^{[19]}$ 采用三嵌段表面活性剂(P123)、正硅酸四乙 酯、磷酸三乙酯、四水硝酸钙和乙醇溶剂混合后喷 雾干燥, 成功制备出球形介孔 $\mathrm{BG}$ 微球, 颗粒粒径为 $1 \mathrm{~nm} 1 \mathrm{~mm}$, 表现出良好的体外诱导磷灰石沉积能 力。 $A r c o s$ 等 ${ }^{[20]}$ 采用不同的结构导向剂(P123、CTAB、 F127)与正硅酸四乙酯、磷酸三乙酯、四水硝酸钻和 乙醇溶剂制备前驱体溶液, 喷雾干燥后制备得到不 同孔径的 BG 微球, 发现氧化钙含量的提高有利于 提高其生物活性。虽然上述研究都表明前驱体溶液 结合喷雾干燥方法可制备不同的 BG 微球, 但是前 驱体溶液通常含有大量易燃溶剂 (主要为体积含量 约 $60 \%$ 的乙醇), 前驱体溶液雾化干燥过程需考虑安
全问题而不能长时间连续工作，限制了 $\mathrm{BG}$ 粉体的 连续批量化制备。

因此，为克服高温耗能、制备周期长、原料成 本高、连续批量制备不安全等缺点, 本研究提出以 水溶液作为溶剂, 在酸性条件下制备 BG 前驱体溶 液, 采用喷雾干燥方法快速、连续、可控制备球形 BG 微球, 研究了喷雾干燥过程中不同工艺参数(进 气风量、前驱体溶液浓度和进料速率)对 $\mathrm{BG}$ 微球粒 径的影响; 通过调节原料配比制备不同化学组成的 $\mathrm{BG}$ 微球, 研究了不同化学组成 BG 微球的体外诱导 磷灰石沉积能力。

\section{1 实验方法}

\section{1 材料}

硝酸 $\left(\mathrm{HNO}_{3}, \geqslant 68 \%\right)$ 、正硅酸四乙酯(TEOS, $98 \%$ )、磷酸三乙酯 (TEP, $99.8 \%)$ 、氯化钠 $(\mathrm{NaCl}$, $99.5 \%)$ 、碳酸氢钠 $\left(\mathrm{NaHCO}_{3}, 99.8 \%\right)$ 、氯化钾 $(\mathrm{KCl}$, $99.8 \%)$ 、磷酸氢二钾 $\left(\mathrm{K}_{2} \mathrm{HPO}_{4} \cdot 3 \mathrm{H}_{2} \mathrm{O}, 99 \%\right)$ 、氯化镁 $\left(\mathrm{MgCl}_{2} \bullet 6 \mathrm{H}_{2} \mathrm{O}, 98 \%\right)$ 、盐酸 $(\mathrm{HCl}, 36 \% \sim 38 \%)$ 、氯化钻 $\left(\mathrm{CaCl}_{2}, 96 \%\right)$ 、硫酸钠 $\left(\mathrm{Na}_{2} \mathrm{SO}_{4}, 99 \%\right)$ 和三羟基氨基 甲烷 $\left(\mathrm{NH}_{2} \mathrm{C}\left(\mathrm{CH}_{2} \mathrm{OH}\right)_{3}, 99 \%\right)$ 购自中国国药化学试剂 有限公司 (中国上海); 硝酸钙 $\left(\mathrm{Ca}\left(\mathrm{NO}_{3}\right)_{2} \bullet 4 \mathrm{H}_{2} \mathrm{O}, 99 \%\right)$ 购自天津市大茂化学检测厂。所有化学药品未经进 一步纯化。

\section{2 制备方法}

\subsection{1 制备前驱体溶液}

室温下, 取一定量的去离子水, 滴加硝酸调节 水溶液至 $\mathrm{pH}$ 为 2 , 接着往水溶液中加入 TEOS 并磁 力摚拌至溶液透明澄清 ${ }^{[21]}$, 然后将 TEP 加入上述澄 清溶液并搅拌 $30 \mathrm{~min}$, 最后加入四水合硝酸钙 $(\mathrm{CaNT})$ 并摚拌 $20 \mathrm{~min}$, 得到澄清的前驱体溶液备用。配制 的不同浓度前驱体溶液如表 1 所示, 不同化学组成 的前驱体溶液如表 2 所示。

\subsection{2 制备 BG 微球}

采用 Buchi-290 喷雾干燥仪 (喷头直径 $2.8 \mathrm{~mm}$ ) 喷雾干燥 $\mathrm{BG}$ 前驱体溶液制备 $\mathrm{BG}$ 微球。固定设置 仪器的循环率为 $100 \%$, 进口温度为 $220{ }^{\circ} \mathrm{C}$ 。前驱体 
溶液浓度分别为 $5 \mathrm{wt} \% 、 15 \mathrm{wt} \% 、 25 \mathrm{wt} \%$ 和 $35 \mathrm{wt} \%$; 进 气风量为 $283 、 439 、 667$ 和 $1052 \mathrm{~L} / \mathrm{h}$; 进料速率为 1.5、3、4.5 和 $6 \mathrm{~mL} / \mathrm{min}$ 。将不同喷雾干燥工艺条 件下收集得到的微球置于马弗炉中，从室温开始以 $2{ }^{\circ} \mathrm{C} / \mathrm{min}$ 的速率升温至 $700{ }^{\circ} \mathrm{C}$ 并保温 $5 \mathrm{~h}$, 自然冷 却得到 $\mathrm{BG}$ 微球。

\subsection{3 体外诱导磷灰石沉积}

为研究不同化学组成 $\mathrm{BG}$ 微球的体外生物活性, 本研究采用实验室自制的模拟体液(SBF)进行体外 矿化实验以验证其体外诱导磷灰石沉积能力。SBF 中各无机离子的浓度与人体血浆中各离子的浓度相 近, 根据 Kukubo 方法配制 ${ }^{[22]}$ 。实验将 $\mathrm{BG}$ 微球以 $0.1 \mathrm{~g} / 100 \mathrm{~mL}$ 的比例浸泡 $\mathrm{SBF}$, 静置于 $37{ }^{\circ} \mathrm{C}$ 烘箱中, 分别浸泡 $2 \mathrm{~h} 、 4 \mathrm{~h} 、 8 \mathrm{~h} 、 12 \mathrm{~h} 、 1 \mathrm{~d} 、 2 \mathrm{~d} 、 3 \mathrm{~d} 、 5 \mathrm{~d}$ 和 $7 \mathrm{~d}$ 后取出, 过滤干燥得到矿化的 BG 微球。采用 扫描电镜和附带的能谱仪观察和分析 BG 微球表面 的羟基磷灰石 $(\mathrm{HA})$ 生长情况; 用粉末 $\mathrm{X}$ 射线衍射仪 分析物相组成; 用红外光谱仪分析化学结构变化。

为了研究不同化学组成 $\mathrm{BG}$ 微球对周围 $\mathrm{pH}$ 环 境的影响, 将 $\mathrm{BG}$ 微球以 $0.1 \mathrm{~g} / 100 \mathrm{~mL}$ 的比例浸泡 于 $\mathrm{Tris}-\mathrm{HCl}(\mathrm{pH} 7.4)$ 溶液并静置于 $37{ }^{\circ} \mathrm{C}$ 烘箱中, 分 别浸泡 $2 \mathrm{~h} 、 4 \mathrm{~h} 、 8 \mathrm{~h} 、 12 \mathrm{~h} 、 1 \mathrm{~d} 、 2 \mathrm{~d} 、 3 \mathrm{~d} 、 5 \mathrm{~d}$ 和 $7 \mathrm{~d}$ 后测试溶液的 $\mathrm{pH}$, 三组平行实验同时进行。

\section{3 样品表征}

用扫描电镜(SEM, Zeiss SIGMA300)观察 BG 微 球形貌, 用所附能谱仪(EDS)分析微球元素成分、组
成及分布，另用 SEM 照片配合 Imaje J 软件对 BG 微 球粒径进行统计; 用粉末 X 射线衍射仪(XRD-6100, SHIMADZU)和红外光谱仪(FT-IR, Nicolet 6700)分 别对 BG 微球进行物相和化学结构分析。

\section{2 结果与讨论}

\subsection{BG 微球结构表征}

裧烧处理后 77SBG 微球的 SEM、粉末 XRD 图 谱以及能谱仪分析得到的元素分布如图 1 所示。由 图 1(A)可以看出, 喷雾干燥法得到的 $B G$ 粉体为光 滑的球形颗粒, 粒径分布较窄; 粉末 XRD 图谱显示 BG 粉体只在 $2 \theta=15^{\circ} \sim 35^{\circ}$ 范围有宽化的馒头峰, 表明 $700{ }^{\circ} \mathrm{C}$ 炦烧处理后的 BG 粉体为无定型态(图 1(B)); 能谱仪分析的元素分布图表明 $\mathrm{BG}$ 微球的 $\mathrm{O}, \mathrm{Si}, \mathrm{Ca}$, $\mathrm{P}$ 元素分布均匀(图 1(C))。由此可知, 采用喷雾干燥 $B G$ 前驱体溶液方法可以制备化学组成分布均匀、 粒径分布较窄的无定型态 BG 微球。

表 1 不同质量浓度的前驱体溶液的配制

Table 1 Preparation of precursor solutions with different mass concentrations

\begin{tabular}{ccccc}
\hline Concentration/wt\% & TEOS/g & TEP/g & CaNT/g & DW/g \\
\hline 5 & 26.8 & 2.92 & 5.6 & 670 \\
15 & 26.8 & 2.92 & 5.6 & 200 \\
25 & 26.8 & 2.92 & 5.6 & 105 \\
35 & 26.8 & 2.92 & 5.6 & 65 \\
\hline
\end{tabular}

表 2 不同化学组成 BG 微球的理论、实际化学组成和反应物用量

Table 2 Theoretical and measured chemical composition, amounts of the reactants of BG microspheres

\begin{tabular}{cccccc}
\hline Sample & $\mathrm{SiO}_{2}: \mathrm{CaO}: \mathrm{P}_{2} \mathrm{O}_{5}(\mathrm{Theo})$ & $\mathrm{SiO}_{2}: \mathrm{CaO}: \mathrm{P}_{2} \mathrm{O}_{5}$ (Measured $)$ & $\mathrm{TEOS} / \mathrm{g}$ & $\mathrm{TEP} / \mathrm{g}$ & $\mathrm{CaNT} / \mathrm{g}$ \\
\hline 58SBG & $60: 36: 4$ & $58.6: 35.1: 3.3$ & 25.08 & 2.92 & 17.04 \\
68SBG & $70: 26: 4$ & $67.1: 24.9: 3.2$ & 29.24 & 2.92 & 12.31 \\
$77 \mathrm{SBG}$ & $80: 16: 4$ & $79.5: 14.7: 3.1$ & 33.42 & 2.92 & 7.58 \\
$86 \mathrm{SBG}$ & $90: 6: 4$ & $89.7: 5.1: 3.6$ & 37.59 & 2.92 & 2.84 \\
\hline
\end{tabular}
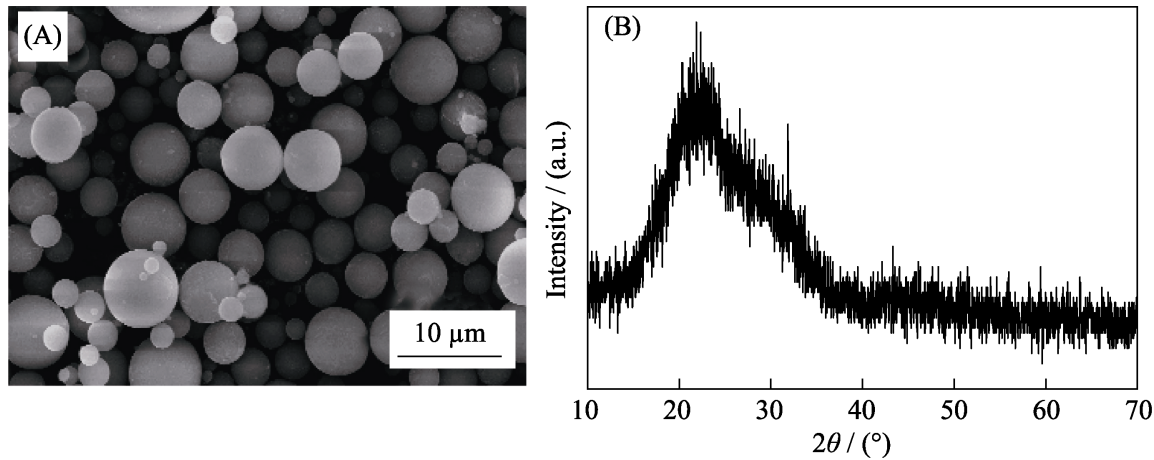

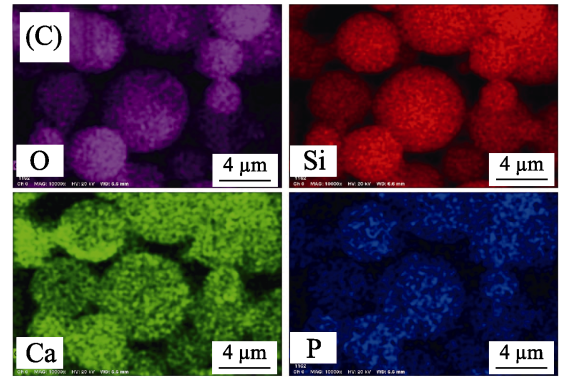

图 1 喷雾干燥法制备的 77SBG 微球的 SEM 照片(A)、粉末 XRD 图谱(B)以及元素分布图(C)

Fig. 1 SEM image (A), XRD pattern (B) and elemental mappings (C) of 77SBG microspheres obtained via spray-drying method 


\section{2 进气风量对 BG 微球粒径的影响}

图 2 是固定进料速率为 $6 \mathrm{~mL} / \mathrm{min}$ 时, 浓度为 $5 \mathrm{wt} \%$ 的前驱体溶液通过调节进气风量分别为 283 、 439、667 和 $1052 \mathrm{~L} / \mathrm{h}$ 时得到 BG 微球的 SEM 照片 和相应的粒径分布直方图。当进气风量为 $283 \mathrm{~L} / \mathrm{h}$ 时，粒径范围为 $5 \sim 30 \mu \mathrm{m}$; 而当进气风量为 $1052 \mathrm{~L} / \mathrm{h}$ 时, 粒径几乎都在 $10 \mu \mathrm{m}$ 以下。说明当进料速率恒 定不变时，单位时间内进气风量越大, $\mathrm{BG}$ 微球的粒 径变小，而且粒径分布变窄。

这是因为单位时间内通过喷嘴风量增大，气流 速率加快, 会将进料的前驱体溶液快速冲散, 从而 使得雾化液滴尺寸变小。另一方面, 气流速率加快将 增加快速转移到溶液流中的能量(动能), 这使得气 液接触面积增大, 被雾化液滴很快达到饱和, 成核
速率加快; 随着成核数量增多, $\mathrm{BG}$ 微球沉积加快, $\mathrm{BG}$ 微球的整体粒径减小，粒径范围也相应变窄 ${ }^{[23-25]}$ 。 因此, 进气风量的增加与雾化流的液滴尺寸成反比, 与最终得到的 $\mathrm{BG}$ 微球的整体粒径大小成反比。

\section{3 前驱体溶液浓度对 BG 微球粒径的影响}

图 3 是进料速率为 $6 \mathrm{~mL} / \mathrm{min}$ 、进气风量为 $667 \mathrm{~L} / \mathrm{h}$ ，前驱体溶液浓度分别为 $5 \mathrm{wt} \% 、 15 \mathrm{wt} \%$ 、 $25 \mathrm{wt} \%$ 和 $35 \mathrm{wt} \%$ 时得到 BG 微球的 SEM 照片和粒径 分布直方图。由图可以看出, 前驱体溶液浓度为 $5 \mathrm{wt} \%$ 时, $\mathrm{BG}$ 微球的粒度范围在 $15 \mu \mathrm{m}$ 以下; 而当前 驱体溶液浓度增加到 $35 \mathrm{wt} \%$ 时, BG 微球的粒度范围 扩大到 $40 \mu \mathrm{m}$ 以下。表明当前驱体溶液浓度增高, $\mathrm{BG}$ 微球整体粒径变大，同时粒径分布变宽。
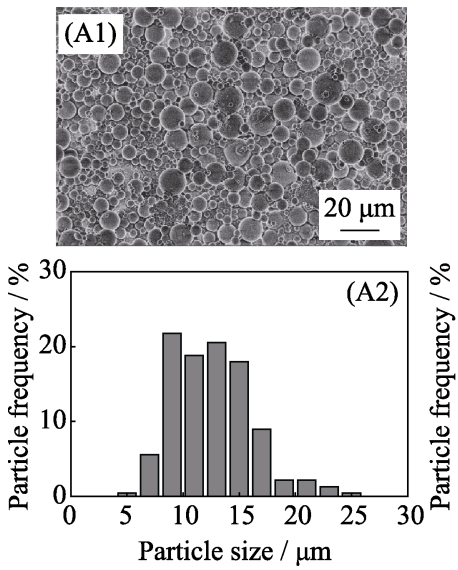
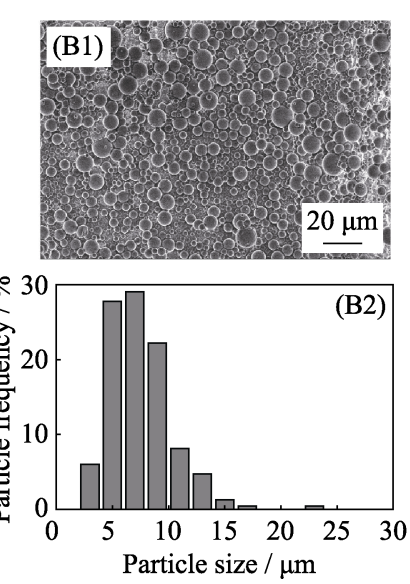
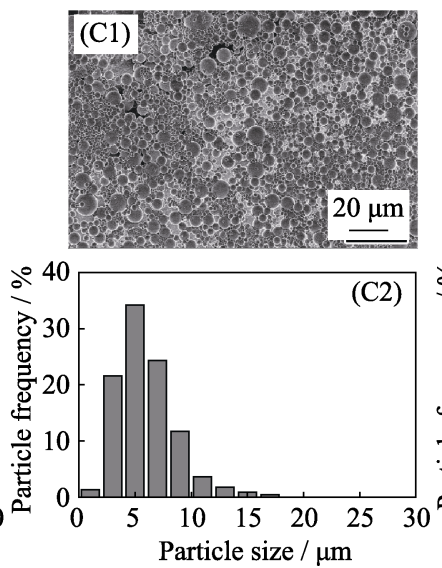

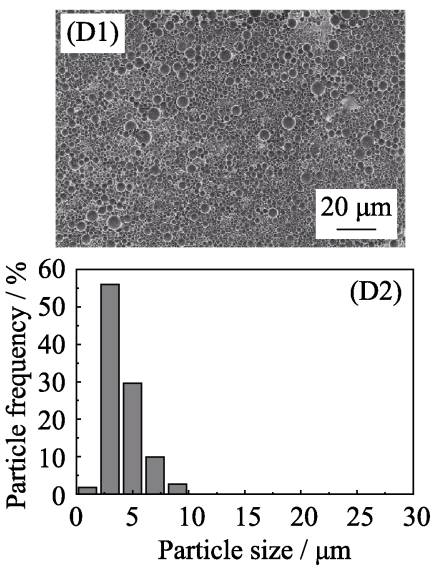

图 2 进料速率为 $6 \mathrm{~mL} / \mathrm{min}$ 时, $5 \mathrm{wt} \%$ 的前驱体溶液在进气风量分别为 $283(\mathrm{~A} 1, \mathrm{~A} 2) 、 439$ (B1, B2)、 $667(\mathrm{C} 1, \mathrm{C} 2)$ 和 $1052 \mathrm{~L} / \mathrm{h}(\mathrm{D} 1, \mathrm{D} 2)$ 时得到的 77SBG 微球的 SEM 照片以及运用 Imaje J 统计得到的粒径直方图

Fig. 2 SEM images and the particle size histogramms obtained by Imaje J statistics of 77SBG microspheres prepared with $5 \mathrm{wt} \%$ precursor solution at inlet air volume of 283 (A1, A2), 439 (B1, B2), $667(\mathrm{C} 1, \mathrm{C} 2)$ and $1052 \mathrm{~L} / \mathrm{h}(\mathrm{D} 1, \mathrm{D} 2)$ when the feed rate was $6 \mathrm{~mL} / \mathrm{min}$
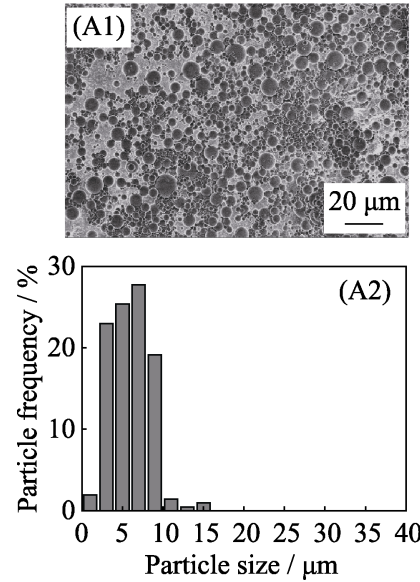
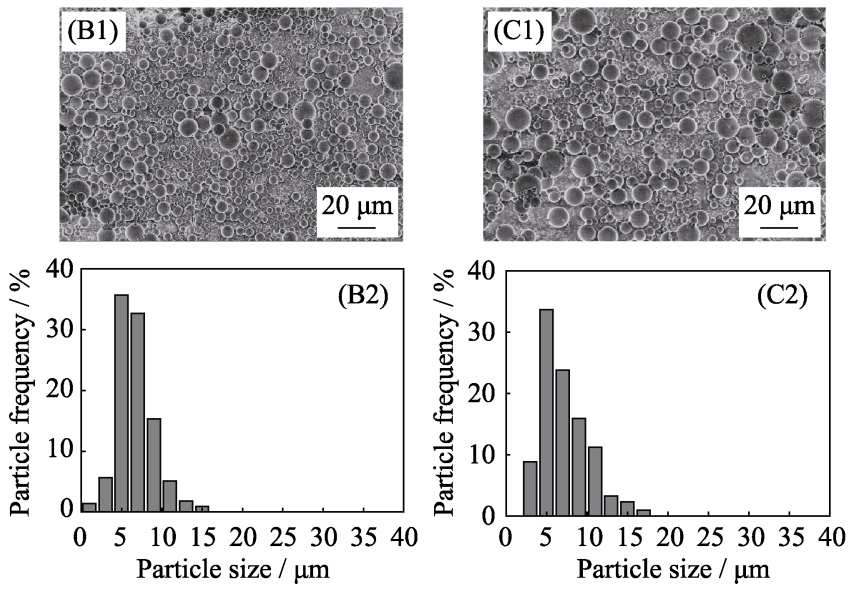

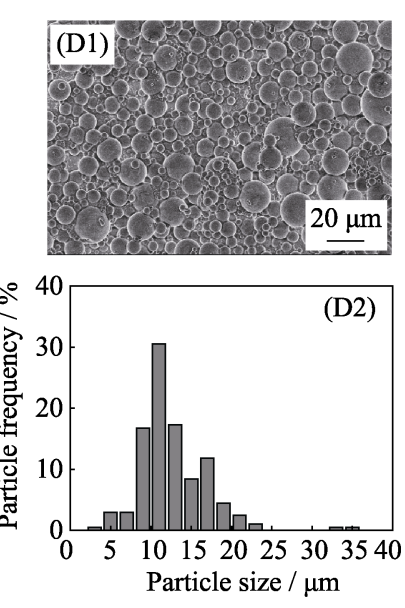

图 3 进气风量为 $667 \mathrm{~L} / \mathrm{h}, 5 \mathrm{wt} \%(\mathrm{~A} 1, \mathrm{~A} 2) 、 15 \mathrm{wt} \%(\mathrm{~B} 1, \mathrm{~B} 2) 、 25 \mathrm{wt} \%(\mathrm{C} 1, \mathrm{C} 2)$ 和 $35 \mathrm{wt} \%(\mathrm{D} 1, \mathrm{D} 2)$

的前驱体溶液在进料为 $6 \mathrm{~mL} / \mathrm{min}$ 时 77SBG 微球的 SEM 照片及运用 Imaje J 统计得到的粒径直方图

Fig. 3 SEM images and the particle size histogramms obtained by Imaje J statistics of 77SBG microspheres prepared with $5 \mathrm{wt} \%(\mathrm{~A} 1, \mathrm{~A} 2), 15 \mathrm{wt} \%(\mathrm{~B} 1, \mathrm{~B} 2), 25 \mathrm{wt} \%(\mathrm{C} 1, \mathrm{C} 2)$ and $35 \mathrm{wt} \%(\mathrm{D} 1, \mathrm{D} 2)$ precursor solutions at the feed rate of $6 \mathrm{~mL} / \mathrm{min}$ when the inlet air volume was $667 \mathrm{~L} / \mathrm{h}$ 
当其他工艺参数不变时, 前驱体溶液浓度增加 造成溶液粘度增大, 使得雾化液滴变大, 导致气液 接触面积缩小, 传热效率降低。因此, 前驱体溶液浓 度增大, $\mathrm{BG}$ 微球的粒径变大且粒度分布也变宽。前 驱体溶液浓度增大, $\mathrm{BG}$ 微球会出现小颗粒团聚附着 在大颗粒表面的现象, 这可能由于溶液粘度增大使 得雾化液滴不均匀所致。

另一方面, 从溶剂去离子水的含量分析, 当其 他工艺参数不变时, 前驱体溶液浓度越小, 意味着 前驱体溶液中的去离子水含量越大。喷雾干燥形成 BG 微球的球形形貌很大程度上取决于溶液的表面 张力 ${ }^{[26]}$ 。在喷雾干燥过程中, 每种原料都会降低雾 化液滴的表面张力, 从而影响最终颗粒的形貌。相 反地, 去离子水具有大的表面张力 $(72.7 \mathrm{mN} / \mathrm{m})$, 高 含量的水会增加雾化液滴整体的表面张力而有利于 形成 BG 微球的球形形貌。因此, 去离子水含量越 高, 喷雾干燥过程中雾化液滴的表面张力越大, 雾 化液滴干燥固化成颗粒时发生的变形越小, 使得形 成的 BG 微球球形度越好, 整体颗粒变小且粒径分 布也会变窄(颗粒更均匀)。

\section{4 进料速率对 BG 微球粒径的影响}

图 4 是前驱体溶液浓度为 $5 \mathrm{wt} \%$ 、进气风量为 $1052 \mathrm{~L} / \mathrm{h}$, 调节进料速率分别为 $1.5 、 3 、 4.5$ 和 $6 \mathrm{~mL} / \mathrm{min}$ 时得到 BG 微球的 SEM 照片和粒径分布 直方图。当进料速率增加时, 得到的 BG 微球的粒 径范围主要为 $10 \mu \mathrm{m}$ 以下, 但是通过 Imaje $\mathrm{J}$ 统计的 粒度分布直方图可以看出, $\mathrm{BG}$ 微球的中间粒径会随 着进料速率的增加而略有增大。这是因为在喷雾干 燥过程中, 进气风量不变, 增大进料速率意味着单
位时间内需要更多能量将前驱体溶液雾化成液滴并 干燥。所以进料速率越大, 雾化液滴的尺寸也会缓 慢增大, 液滴干燥后形成的 $\mathrm{BG}$ 微球的中间粒径也 会略有增大。因此, 喷雾干燥过程中进料速率对 BG 微球的粒径范围影响较小, 但是进料速率增大会 使 BG 微球的中间粒径略有增大。

\section{5 不同化学组成 BG 微球的结构表征}

图 5 是 $58 \mathrm{SBG} 、 68 \mathrm{SBG} 、 77 \mathrm{SBG}$ 和 $86 \mathrm{SBG}$ 微 球在 $700{ }^{\circ} \mathrm{C}$ 炦烧处理后的 SEM 照片。可以看出, 不 同化学组成的前驱体溶液都能采用喷雾干燥方法制 备得到表面光滑的 BG 微球, 并且相同的喷雾干燥 过程得到粒径范围相似的 $\mathrm{BG}$ 微球。图 6 是 58SBG、 $68 \mathrm{SBG} 、 77 \mathrm{SBG}$ 和 $86 \mathrm{SBG}$ 微球在 $700{ }^{\circ} \mathrm{C}$ 㷽烧处理 后的 XRD 图谱和 FT-IR 图谱。由 XRD 图谱可以看 出, $86 \mathrm{SBG}$ 和 $77 \mathrm{SBG}$ 微球为典型的无定型态生物玻 璃, 而 $68 \mathrm{SBG}$ 微球出现了较弱的 $\mathrm{Ca}_{2} \mathrm{SiO}_{4}(\mathrm{PDF} \# 86-$ 0399)物相宽化衍射峰, $58 \mathrm{SBG}$ 则显示出明显的 $\mathrm{Ca}_{2} \mathrm{SiO}_{4}$ 衍射峰, 表明随着 $\mathrm{Ca} / \mathrm{Si}$ 比例的增大, $700{ }^{\circ} \mathrm{C}$ 制烧处理得到的 $\mathrm{BG}$ 微球由完全的无定型态到逐渐 有 $\mathrm{Ca}_{2} \mathrm{SiO}_{4}$ 物相生成。由 FT-IR 图谱可看出, $58 \mathrm{SBG}$ 、 $68 \mathrm{SBG} 、 77 \mathrm{SBG}$ 和 $86 \mathrm{SBG}$ 微球在 800 和 $1083 \mathrm{~cm}^{-1}$ 左右处出现了较强的 $\mathrm{Si}-\mathrm{O}-\mathrm{Si}$ 的伸缩振动峰; 在 $460 \mathrm{~cm}^{-1}$ 左右处出现了 $\mathrm{Si}-\mathrm{O}-\mathrm{Si}$ 的弯曲振动峰; $58 \mathrm{SBG}$ 和 $68 \mathrm{SBG}$ 微球在 $590 \mathrm{~cm}^{-1}$ 左右处出现微弱 的肩峰属于无定型态 $\mathrm{P}-\mathrm{O}$ 的弯曲振动双峰; 从图中 可看出四种组分的 $\mathrm{BG}$ 微球在 $3500 \mathrm{~cm}^{-1}$ 左右处出现 了- $\mathrm{OH}$ 峰, 这是吸收了空气中水分的缘故。另外, $58 \mathrm{SBG}$ 微球在 $1480 \mathrm{~cm}^{-1}$ 处出现了 $\mathrm{CO}_{3}{ }^{2-}$ 的振动峰, 这是因为相对于 $68 \mathrm{SBG} 、 77 \mathrm{SBG} 、 86 \mathrm{SBG}$ 微球,
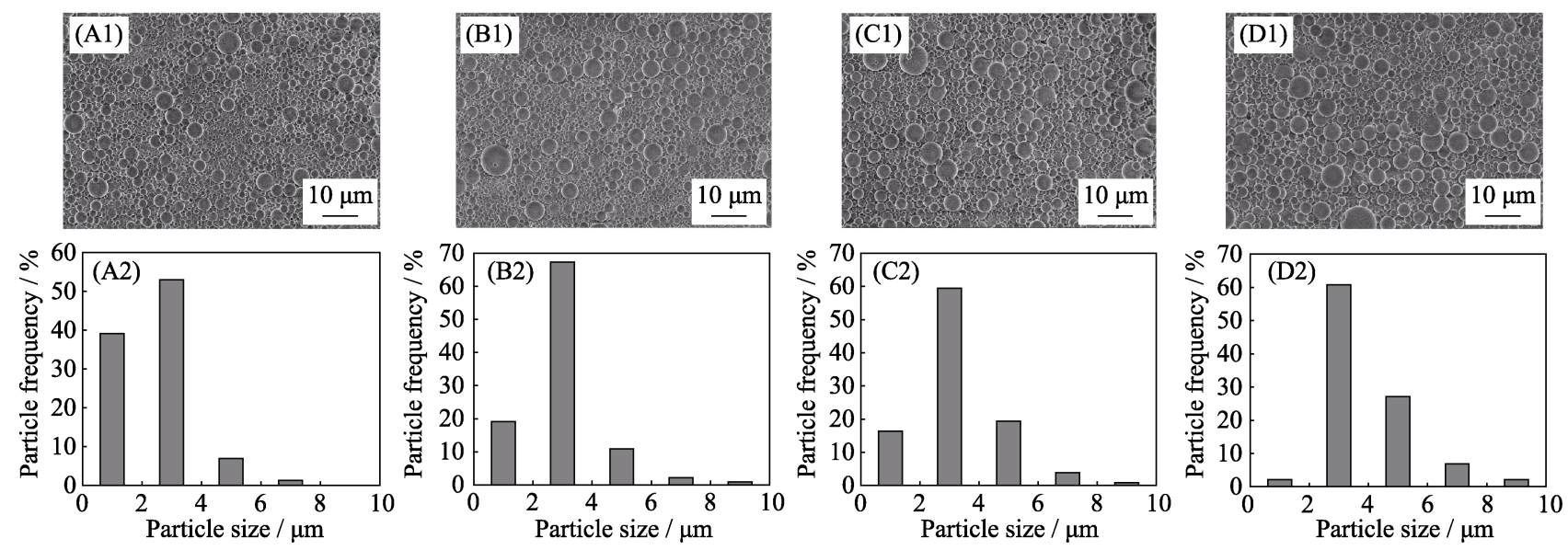

图 4 进气风量为 $1052 \mathrm{~L} / \mathrm{h}$ 时, $5 \mathrm{wt} \%$ 的前驱体溶液在进料速率为 $1.5 \mathrm{~mL} / \mathrm{min}(\mathrm{A} 1, \mathrm{~A} 2) 、 3 \mathrm{~mL} / \mathrm{min}(\mathrm{B} 1, \mathrm{~B} 2)$ 、 $4.5 \mathrm{~mL} / \mathrm{min}(\mathrm{C} 1, \mathrm{C} 2)$ 和 $6 \mathrm{~mL} / \mathrm{min}(\mathrm{D} 1, \mathrm{D} 2)$ 时得到的 $77 \mathrm{SBG}$ 微球的 SEM 照片及运用 Imaje $\mathrm{J}$ 统计得到的粒径直方图

Fig. 4 SEM images and the particle size histogramms obtained by Imaje J statistics of 77SBG microspheres prepared with the $5 \mathrm{wt} \%$ precursor solution at the feed rates of $1.5 \mathrm{~mL} / \mathrm{min}(\mathrm{A} 1, \mathrm{~A} 2), 3 \mathrm{~mL} / \mathrm{min}(\mathrm{B} 1, \mathrm{~B} 2)$, $4.5 \mathrm{~mL} / \mathrm{min}(\mathrm{C} 1, \mathrm{C} 2)$ and $6 \mathrm{~mL} / \mathrm{min}(\mathrm{D} 1, \mathrm{D} 2)$ when the inlet air volume was $1052 \mathrm{~L} / \mathrm{h}$. 

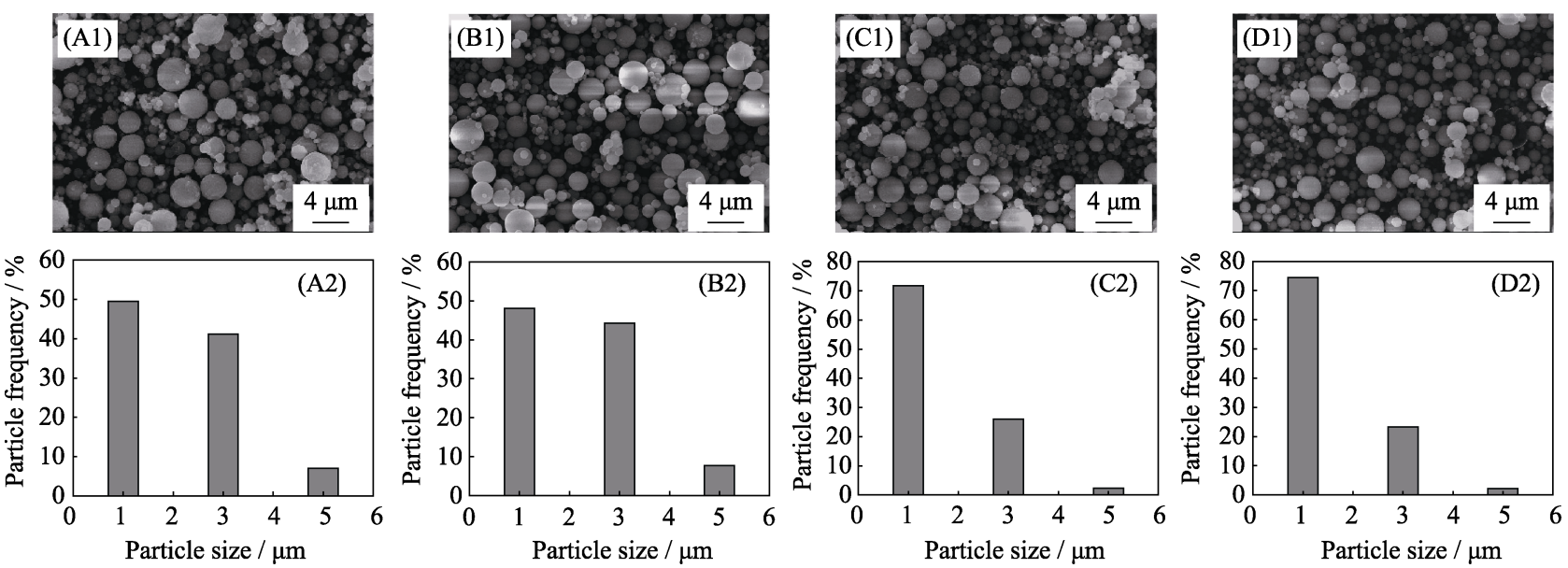

图 5 58SBG(A1, A2)、68SBG(B1, B2)、77SBG(C1, C2)和 $86 \mathrm{SBG}(\mathrm{D} 1, \mathrm{D} 2)$ 微球经 $700{ }^{\circ} \mathrm{C}$ 㷽烧处理后 的 SEM 照片及运用 Imaje $\mathrm{J}$ 统计的粒径分布直方图

Fig. 5 SEM images and the particle size histogramms obtained by Imaje J statistics of 58SBG (A1, A2), 68SBG (B1, B2), 77SBG (C1, C2) and 86SBG (D1, D2) microspheres after calcination at $700{ }^{\circ} \mathrm{C}$
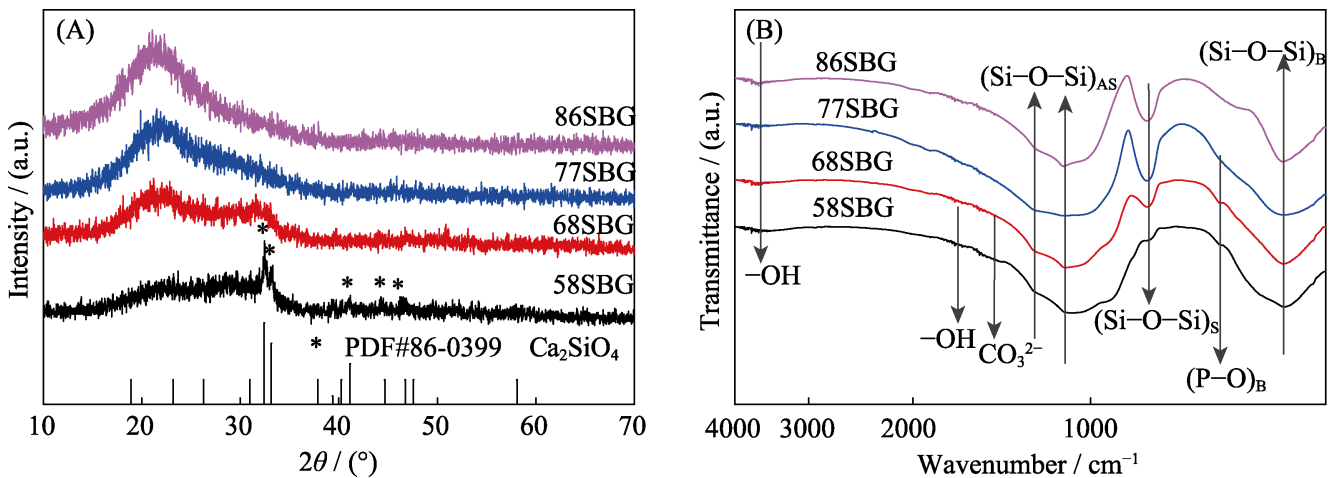

图 $658 \mathrm{SBG} 、 68 \mathrm{SBG} 、 77 \mathrm{SSBG}$ 和 $86 \mathrm{SBG}$ 微球在 $700{ }^{\circ} \mathrm{C}$ 煅烧处理后的广角 XRD 图谱(A)和 FT-IR 谱图(B)

Fig. 6 Wide-angle XRD patterns (A) and FT-IR spectra (B) of 58SBG, 68SBG, 77SSBG and $86 \mathrm{SBG}$ microspheres after calcination at $700{ }^{\circ} \mathrm{C}$

$58 \mathrm{SBG}$ 微球氧化钙含量最大, 表征时暴露在空气中 更容易吸收水分和二氧化碳的缘故。

\section{6 不同化学组成 BG 微球的体外诱导磷灰石 沉积能力}

图 7 是 58SBG、68SBG、77SBG 和 $86 \mathrm{SBG}$ 微 球在 SBF 中浸泡 $7 \mathrm{~d}$ 后的 SEM 照片和 EDS 分析图 谱。由 SEM 照片可以看出, 原本光滑的 BG 微球表 面都覆盖了一层厚厚的沉积物。根据 EDS 能谱分析 得到 $58 \mathrm{SBG} 、 68 \mathrm{SBG} 、 77 \mathrm{SBG}$ 和 $86 \mathrm{SBG}$ 微球的 $\mathrm{Ca} / \mathrm{P}$ 比值分别为 $2.048 、 1.597 、 1.520$ 和 1.712 , 这些比值 都较接近于羟基磷灰石的 $\mathrm{Ca} / \mathrm{P}=1.67$, 表明 $\mathrm{BG}$ 微球 表面的沉积物为羟基磷灰石。因此, 喷雾干燥前驱 体溶液制备的 BG 微球具有良好的体外诱导磷灰石 沉积能力, 主要是因为快速加热和快速冷却的喷雾 干燥过程使得制备的 BG 颗粒的表面保留了更多的 亚稳硅氧烷基团 ${ }^{[18]}$, 而这些基团则充当成核位点有 助于羟基磷灰石形成 ${ }^{[9]}$ 。
图 8 为 $58 \mathrm{SBG} 、 68 \mathrm{SBG} 、 77 \mathrm{SBG}$ 和 $86 \mathrm{SBG}$ 微 球在 SBF 中浸泡不同时间前后的 XRD 图谱。可以 看出, 不同化学组成的 $\mathrm{BG}$ 微球在 SBF 中浸泡不同 时间后在 $2 \theta=25.8^{\circ} 、 31.7^{\circ} 、 39^{\circ}$ 和 $47^{\circ}$ 处都出现了羟 基磷灰石的特征衍射峰(PDF\#09-0432), 表明不同 化学组成的 $\mathrm{BG}$ 微球都具有体外诱导磷灰石沉积能 力。另一方面, 不同化学组成的 BG 微球矿化生成 羟基磷灰石的能力有所不同。其中, $86 \mathrm{SBG}$ 微球在 矿化 $3 \mathrm{~d}$ 后出现明显的羟基磷灰石特征衍射峰, 而 58SBG、68SBG 和 77SBG 微球则在矿化 $4 \mathrm{~h}$ 后就 出现了羟基磷灰石的特征衍射峰, 并且随着矿化时 间延长, 羟基磷灰石的特征衍射峰强度逐渐增 强; $58 \mathrm{SBG}$ 微球在浸泡 $5 \mathrm{~d}$ 后出现了较强的 $\mathrm{CaCO}_{3}$ 物相衍射峰 ${ }^{[27]}$, 这归因于 $58 \mathrm{SBG}$ 微球表面较高的 $\mathrm{Ca} / \mathrm{P}$ 比使 $58 \mathrm{SBG}$ 微球溶于 $\mathrm{SBF}$ 后的钙离子溶出较 多, 在 SBF 中矿化形成羟基磷灰石的同时还形成了 $\mathrm{CaCO}_{3}$ 。 

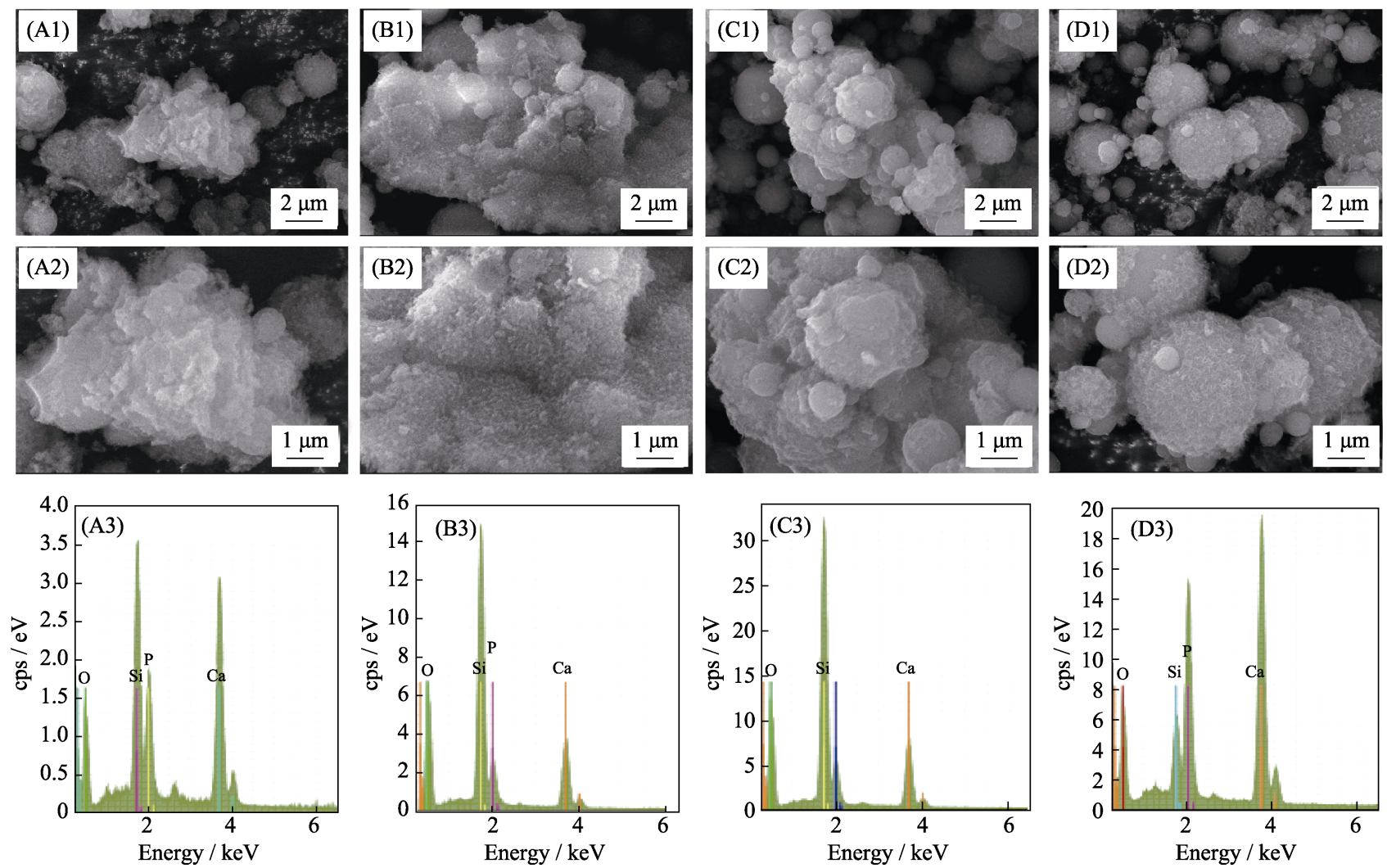

图 $758 \mathrm{SBG}(\mathrm{A}) 、 68 \mathrm{SBG}(\mathrm{B}) 、 77 \mathrm{SSBG}(\mathrm{C})$ 和 $86 \mathrm{SBG}(\mathrm{D})$ 微球在 $\mathrm{SBF}$ 中浸泡 $7 \mathrm{~d}$ 后的 $\mathrm{SEM}$ 照片和 $\mathrm{EDS}$ 分析图谱 Fig. 7 SEM images and EDS analysis of 58SBG (A), 68SBG (B), 77SSBG (C), and 86SBG (D) microspheres after soaking in SBF for $7 \mathrm{~d}$
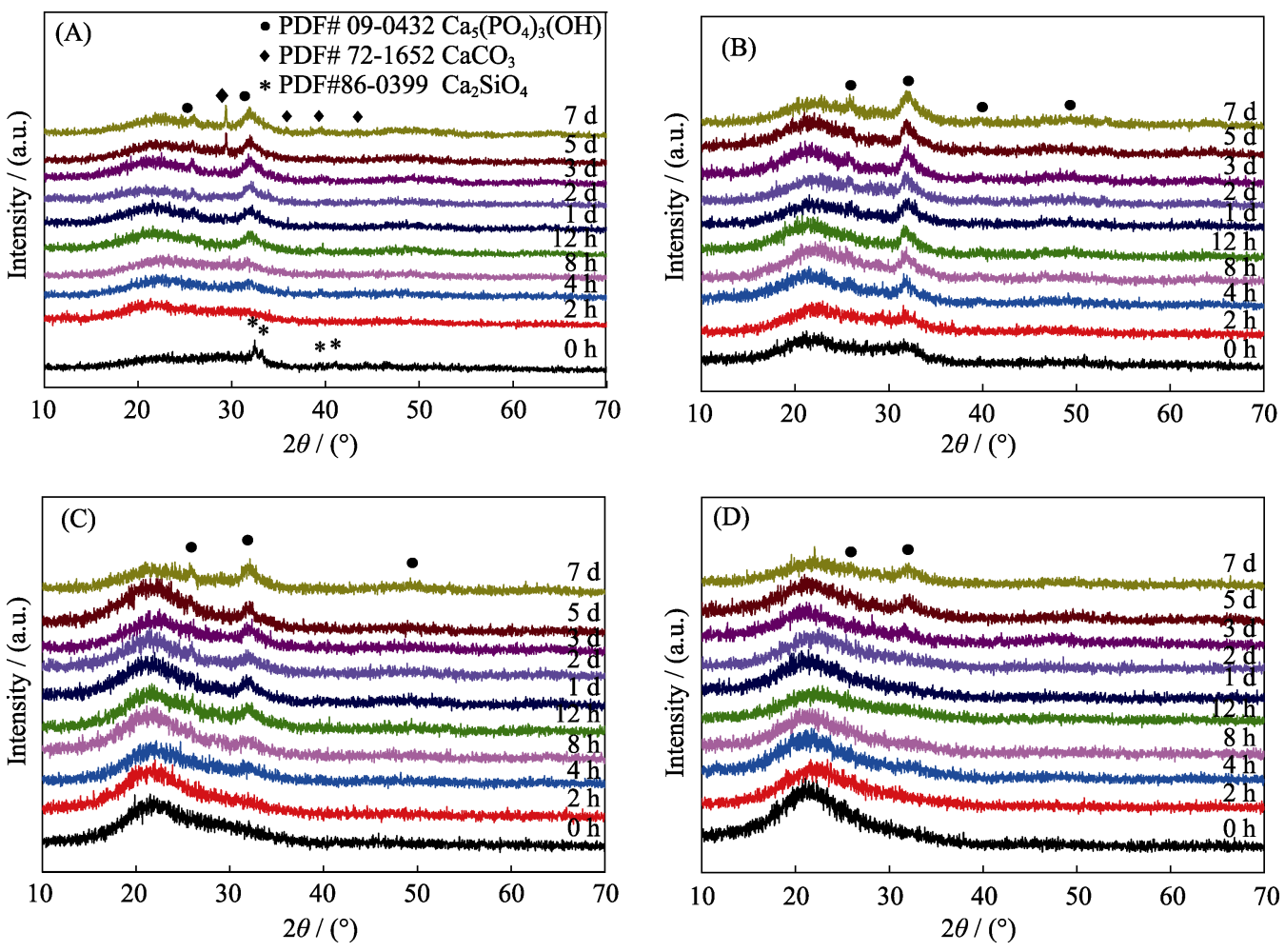

图 8 58SBG(A)、68SBG(B)、77SSBG(C) 和 $86 \mathrm{SBG}(\mathrm{D})$ 微球在 SBF 中浸泡不同时间前后的广角 XRD 图谱

Fig. 8 Wide-angle XRD patterns of 58SBG (A), 68SBG (B), 77SSBG (C) and 86SBG (D) microspheres before and after soaking in SBF for different periods 
图 9 为 $58 \mathrm{SBG} 、 68 \mathrm{SBG} 、 77 \mathrm{SBG}$ 和 $86 \mathrm{SBG}$ 微 球在 SBF 中浸泡不同时间前后的 FT-IR 谱图。可以 看出, 不同化学组成的 $\mathrm{BG}$ 微球在 SBF 中浸泡不同 时间后，在 560 和 $610 \mathrm{~cm}^{-1}$ 左右都出现了晶态的 $\mathrm{P}-\mathrm{O}$ 弯曲振动双峰; 在 $1033 \mathrm{~cm}^{-1}$ 附近出现 $\mathrm{P}-\mathrm{O}$ 伸 缩振动峰, 而且随着矿化时间延长, 振动峰强度也 逐渐增强, 表明 $\mathrm{BG}$ 微球在矿化过程中, 羟基磷灰石 在 BG 微球表面的逐渐形成和晶化。另一方面, 从图 9 中还可以观察到 58SBG 微球浸泡 SBF 后在 1410 和 $1450 \mathrm{~cm}^{-1}$ 附近出现 $\mathrm{CO}_{3}{ }^{2-}$ 振动峰 ${ }^{[28-29]}$, 随着浸泡时 间延长, 其振动峰更明显; 不仅如此, 在浸泡 $2 \mathrm{~d}$ 时, 明显观察到在 $870 \mathrm{~cm}^{-1}$ 左右也出现 $\mathrm{CO}_{3}{ }^{2-}$ 中 $\mathrm{C}-\mathrm{O}$ 振 动峰, 这表明 $58 \mathrm{SBG}$ 微球表面矿化形成碳酸差基磷 灰石 ${ }^{[30-31]}$; 同理也可看出 $68 \mathrm{SBG}$ 和 $77 \mathrm{SBG}$ 微球在 浸泡 $3 \mathrm{~d}$ 时, 在 1410 和 $1450 \mathrm{~cm}^{-1}$ 附近也出现比较 微弱的 $\mathrm{CO}_{3}{ }^{2-}$ 振动峰, 表明在 $68 \mathrm{SBG}$ 和 $77 \mathrm{SBG}$ 微球 的表面也生成很少量的碳酸着基磷灰石。

图 10 是 $58 \mathrm{SBG} 、 68 \mathrm{SBG} 、 77 \mathrm{SBG}$ 和 $86 \mathrm{SBG}$ 微 球浸泡 Tris- $\mathrm{HCl}$ 溶液中的 $\mathrm{pH}$ 变化曲线。由图可以看 出, 不同组成的 $\mathrm{BG}$ 微球浸泡 Tris-HCl 溶液后, 其溶 液 $\mathrm{pH}$ 随着浸泡时间的延长而升高，且浸泡小于 $24 \mathrm{~h}$ 的 $\mathrm{pH}$ 均增大较快, 随后缓慢增大, 最终 $\mathrm{pH}$ 分别稳 定在 7.63、7.73、8.49 和 9.29 左右。其中, $58 \mathrm{SBG}$
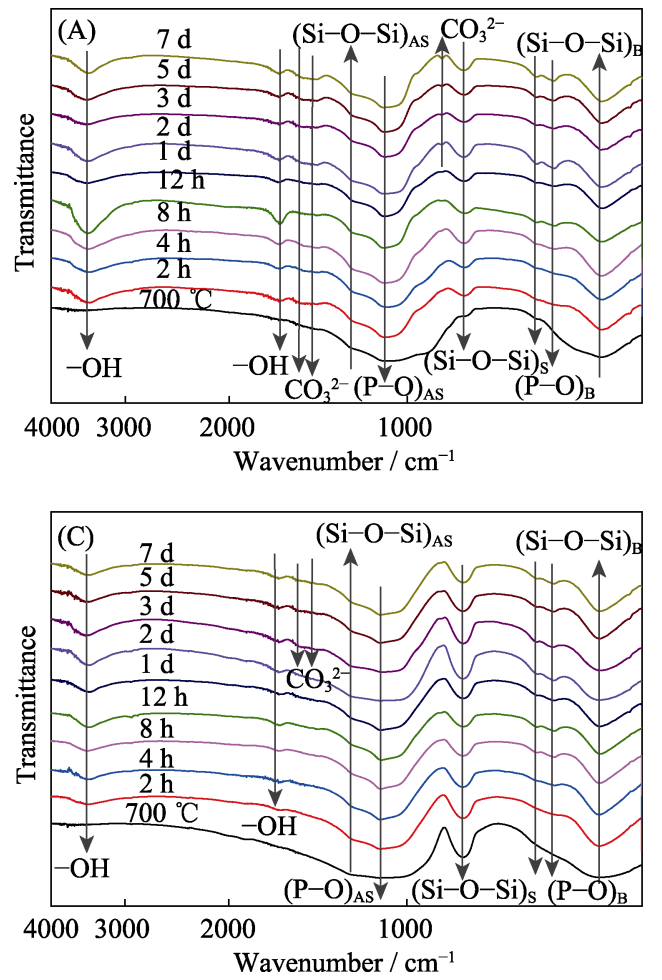

微球在浸泡前 $24 \mathrm{~h}$ 增加最快, 最终达到的 $\mathrm{pH}$ 最高。 这主要因为 $58 \mathrm{SBG}$ 微球的 $\mathrm{Ca}$ 含量最大, 当 $\mathrm{BG}$ 微 球浸泡在 Tris- $\mathrm{HCl}$ 溶液中后, 微球会发生降解并溶解 出大量的 $\mathrm{Ca}^{2+}$, 而 $\mathrm{Ca}^{2+}$ 会在溶液中发生水化反应产 生 $\mathrm{OH}^{-}$, 从而使溶液 $\mathrm{pH}$ 升高而呈现碱性环境 ${ }^{[32]}$ 。

\section{3 结论}

本研究以正硅酸四乙酯、磷酸三乙酯和四水硝 酸钙为原料, 采用喷雾干燥前驱体溶液方法可控制 备出球形、组成分布均匀且无定型的 $B G$ 微球。通 过调节喷雾干燥过程中的工艺参数(进气风量、前驱 体溶液浓度和进料速率)可得到粒径可控的 $\mathrm{BG}$ 微 球。进气风量增大, $\mathrm{BG}$ 微球的整体粒径减小、粒径 分布变窄; 前驱体溶液浓度增大, $\mathrm{BG}$ 微球的整体粒 径增大、粒径分布变宽; 进料速率增大, $\mathrm{BG}$ 微球的 整体粒径略有增大、粒径分布基本不变。另一方面, 喷雾干燥方法还可以制备不同化学组成的 BG 微球, 且都具有良好的诱导磷灰石沉积能力, $B G$ 微球中钙 含量增加有利于提高 $\mathrm{BG}$ 微球的体外诱导磷灰石沉 积能力。因此, 本研究提出的喷雾干燥法制备 $\mathrm{BG}$ 微球可实现粒径、化学组成可控并有望快速、批量 制备 BG 微球。
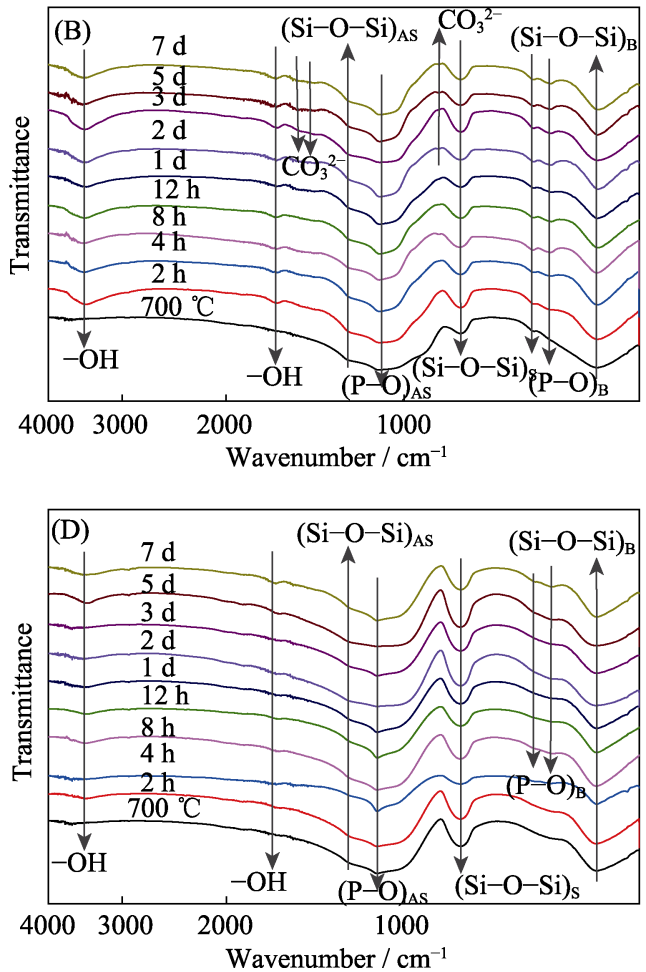

图 $958 \mathrm{SBG}(\mathrm{A}) 、 68 \mathrm{SBG}(\mathrm{B}) 、 77 \mathrm{SSBG}(\mathrm{C})$ 和 $86 \mathrm{SBG}(\mathrm{D})$ 微球在 $\mathrm{SBF}$ 中浸泡不同时间前后的 FT-IR 谱图

Fig. 9 FTIR spectra of 58SBG (A), 68SBG (B), 77SBG (C) and 86SBG (D) microspheres before and after soaking in SBF for different periods 


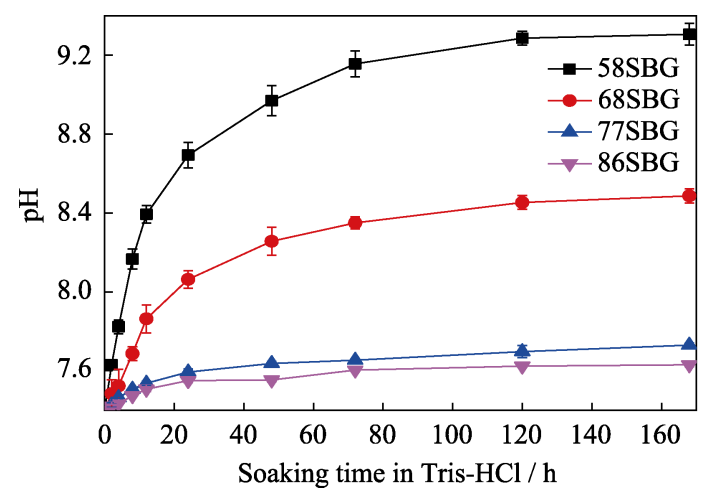

图 10 58SBG、68SBG、77SBG 和 $86 \mathrm{SBG}$ 微球在 Tris- $\mathrm{HCl}$ 溶液中浸泡不同时间的 $\mathrm{pH}$ 变化曲线

Fig. $10 \mathrm{pH}$ changes of Tris- $\mathrm{HCl}$ solutions after 58SBG, 68SBG, 77SBG and 86SBG microspheres soaking in Tris- $\mathrm{HCl}$ for different periods

\section{参考文献:}

[1] JONES J R. Review of bioactive glass: From Hench to hybrids. Acta Biomaterialia, 2015, 23: S53-S82.

[2] HULBERT S F, HENCH L L, FORBERS D, et al. The story of bioceramics. Ceramics International, 1982, 8(4): 131-140.

[3] FU S, HU H, CHEN J, et al. Silicone resin derived larnite/C scaffolds via 3D printing for potential tumor therapy and bone regeneration. Chemical Engineering Journal, 2020, 382: 122928.

[4] FU S Y, ZHU M, ZHU Y F. Organosilicon polymer-derived ceramics: an overview. Journal of Advanced Ceramics, 2019, 8(4): 457-478.

[5] ZHANG J J, CHEN X, LIN C, et al. Preparation of nanoscale bioactive glasses by addition of PEG as surface dispersion agent. Bulletin of the Chinese Ceramic Society, 2010, 29(2): 257-261.

[6] CHEN X, MENG Y, LI Y. Investigation on bio-mineralization of melt and Sol-Gel derived bioactive glasses. Applied Surface Science, 2008, 255(2): 562-564.

[7] ZHONG J P, GREENSPAN D C. Processing and properties of SolGel bioactive glasses. Journal of Biomedical Materical Research, 2000, 53(6): 694-701.

[8] PAN S, YIN J, YU L, et al. 2D MXene-integrated 3D-printing scaffolds for augmented osteosarcoma phototherapy and accelerated tissue reconstruction. Advanced Science, 2020, 7: 1901551.

[9] DU X, WEI D, HUANG L, et al. 3D printing of mesoporous bioactive glass/silk fibroin composite scafolds for bone tissue engineering. Materials Science \& Engineering C, 2019, 103: 109731.

[10] PONTIROLI L, DADKHAH M, NOVAJRA G, et al. An aerosolspray-assisted approach to produce mesoporous bioactive glass microspheres under mild acidic aqueous conditions. Materials Letters, 2017, 190: 111-114.

[11] YAMAUCHI Y, SUZUKI N, GUPTA P, et al. Aerosol-assisted synthesis of mesoporous organosilica microspheres with controlled organic contents. Science and Technology of Advanced Materials, 2009, 10(2): 025005-025009.

[12] FIORILLI S, TALLIA F, Pontiroli L, et al. Spray-dried mesoprous silica spheres functionalized with carboxylic groups. Materials Letters, 2013, 108: 118-121.

[13] LIU W J, WU W D, SELOMULYA C, et al. A single step assembly of uniform microparticles for controlled release applications. Soft
Matter, 2011, 7(7): 3323.

[14] WU W D, AMELIA R, HAO N, et al. Assembly of uniform photoluminescent microcomposites using a novel micro-fluidic-jetspray-dryer. AIChE Journal, 2011, 57: 2726-2737.

[15] AMELIA R, WU W D, CASHION J, et al. Microfluidic spray drying as a versatile assembly route of functional particles. Chemical Engineering Science, 2011, 66(22): 5531-5540

[16] AMELIA R, WU W D, CHEN X D, et al. Assembly of magnetic microcomposites from low $\mathrm{pH}$ precursors using a novel micro-fluidic-jet-spray-dryer. Chemical Engineering Research \& Design, 2012, 90(1): 150-157.

[17] LIU W J, WU W D, SELOMULYA C, et al. Facile spray-drying assembly of uniform microencapsulates with tunable core-shell structures and controlled release properties. Langmuir, 2011, 27(21): 12910-12915.

[18] SHIH S J, Chou Y J, BORISENKO K B. Preparation method: structure-bioactivity correlation in mesoporous bioactive glass. Journal of Nanoparticle Research, 2013, 15(6): 1763.

[19] OSTOMEL T, SHI Q, TSUNG C K, et al. Spherical bioactive glass with enhanced rates of hydroxyapatite deposition and hemostatic activity. Small, 2006, 2(11): 1261-1265.

[20] ARCOS D, LOPEZ-NORIEGA A, RUIZ-HERNA NDEZ E, et al. Ordered mesoporous microspheres for bone grafting and drug delivery. Chemistry of Materials, 2009, 21(6): 1000-1009.

[21] WU W D, LIU W J, SELOMULYA C, et al. On spray drying of uniform silica-based microencapsulates for controlled release. Soft Matter, 2011, 7(24): 11416-11424.

[22] KOKUBO T, TAKADAMA H. How useful is SBF in predicting in vivo bone bioactivity? Biomaterials, 2006, 27(15): 2907-2915.

[23] MASTERS K. Spray Drying Handbook, fifth ed. Harlow: Longman Scientific and Technical, 1991, 15(3): 344-345.

[24] LAVERNIA E J, WU Y. Spray atomization and deposition. Journal of Materials Processing Technology, 2000: 278-279

[25] NUKIYAMA S, TANASAWA Y. Experiments on the atomization of liquids in an airstream. Transactions of the Canadian Society for Mechanical Engineering, 1940: S7.

[26] IDE M, WALLAERT E, DRIESSCHE I V, et al. Spherical mesoporous silica particles by spray drying: doubling the retention factor of HPLC columns. Microporous \& Mesoporous Materials, 2011, 142(1): 282-291

[27] FUJITA Y, YAMAMURO T, NAKAMURA T, et al. The bonding behavior of calcite to bone. Journal of Biomedical Materical Research, 1991, 25: 991-1003.

[28] ŚLÓSARCZYK A, PASZKIEWICZ Z, PALUSZKIEWICZ C. FTIR and XRD evaluation of carbonated hydroxyapatite powders synthesized by wet methods. Journal of Molecular Structure, 2005, 744: 657-661.

[29] MEYER J L, FOWLER B O. Lattice defects in nonstoichiometric calcium hydroxylapatites: a chemical approach. Inorganic Chemistry, 1982, 21(8): 3029-3035.

[30] MIAO X, TAN L P, TAN L S, et al. Porous calcium phosphate ceramics modified with PLGA-bioactive glass. Materials Science and Engineering, 2007, 27(2): 274-279.

[31] RICHARD M D, ALDO R B. Effect of particulate bioactive glasses on human macrophages and monocytes in vitro. Journal of Biomedical Materical Research Part A, 2005, 73A(1): 73-79.

[32] LUTZ-CHRISTIAN G, BOCCACCINI A R. Review - bioactive glass and glass-ceramic scaffolds for bone tissue engineering. Materials, 2010, 3(7): 3867-3910. 